\title{
Sıcaklık Şoku Uygulaması ile Triploid Karadeniz Alabalığı (Salmo trutta labrax) Üretimi ve Kuluçka Performansının Belirlenmesi ["]
}

\author{
Fatma DELIHASAN SONAY ${ }^{1^{*}}$ Nadir BAŞÇINAR ${ }^{2}$ Süleyman AKHAN ${ }^{3}$ \\ ${ }^{1}$ Recep Tayyip Erdoğan Üniversitesi, Su Ürünleri Fakültesi, Yetiştiricilik Bölümü, Rize, Türkiye \\ ${ }^{2}$ Karadeniz Teknik Üniversitesi, Sürmene Deniz Bilimleri Fakültesi, Balıkçıllk Teknolojisi Mühendisliği Bölümü, Trabzon, Türkiye \\ ${ }^{3}$ Antalya Üniversitesi, Su Ürünleri Fakültesi, Yetiştiricilik Bölümü, Antalya, Türkiye
}

Atıf yapmak için: Sonay Delihasan, F., Baş̧ınar, N. \& Akhan, S. (2021). Sıcaklık Şoku Uygulaması ile Triploid Karadeniz Alabalığı (Salmo trutta labrax) Üretimi ve Kuluçka Performansının Belirlenmesi. Anadolu Çev. ve Hay. Dergisi, 6(4), 635-641.

How to cite: Sonay Delihasan, F., Başçınar, N. \& Akhan, S. (2021). Triploid Black Sea Trout (Salmo trutta labrax) Production by Using Heat Shocks and Determination of Incubation Performance. J. Anatolian Env. and Anim. Sciences, 6(4), 635-641.

D: https://orcid.org/0000-0002-0511-4782 iD : https://orcid.org/0000-0002-5295-0871 (iD) : https://orcid.org/0000-0002-9122-1495

*Sorumlu yazarın: Fatma DELIHASAN SONAY

Recep Tayyip Erdoğan Üniversitesi, Su Ürünleri Fakültesi, 53100 Rize-Türkiye 凶: fatma.delihasan@erdogan.edu.tr
Öz: Bu çalışmada, triploid Karadeniz alabalığı (Salmo trutta labrax) üretmek için termal şok protokolünün optimizasyonu amaçlanmıştır. Triploidizasyon işlemi için yumurtalar döllendikten sonra 10,15 ve 20 . dakikalarda dört farklı sicaklıkta $\left(26,28,30\right.$ ve $\left.32^{\circ} \mathrm{C}\right)$ şok uygulanmıştır. Deneysel gruplarda ploidi oranı NOR (Nükleolar Organizatör Bölgeleri) boyama ve eritrosit büyüklügü yöntemleri ile belirlenmiştir. En yüksek triploid oranları, döllendikten 15 dakika sonra 10 dakika sicaklık şoku uygulamasıly $32{ }^{\circ} \mathrm{C}$ 'de $\% 86,15$ ve $28{ }^{\circ} \mathrm{C}$ 'de $\% 81,26$ olarak tespit edilmiştir. Kuluçka randıman $128{ }^{\circ} \mathrm{C}$ sıcaklık şoku grubunda daha başarılı bulunmuştur. Çalışmadan elde edilen bulguların, Karadeniz alabalığında triploid stok üretimi için kullanılabileceği kanaatine varılmıştır.

\section{Triploid Black Sea Trout (Salmo trutta labrax) Production by Using Heat Shocks and Determination of Incubation Performance ${ }^{[*]}$}

*Corresponding author's: Fatma DELIHASAN SONAY Recep Tayyip Erdogan University, Faculty of Fisheries, 53100, Rize, Turkey 凶: fatma.delihasan@erdogan.edu.tr

\begin{abstract}
In this study, optimization of thermal shock protocol for triploid Black Sea trout (Salmo trutta labrax) production was aimed. In total, four different temperature regimes $(26,28$, 30 , and $32^{\circ} \mathrm{C}$ ) were applied to fertilized eggs following fertilization at $10^{\text {th }}, 15^{\text {th }}$, and $20^{\text {th }}$ minutes for the triploidization process. Ploidy rate in experimental groups was determined by NOR (Nucleolar Organizer Regions) and measures of erythrocyte size. The highest triploid rates were determined to be $86.15 \%$ at $32^{\circ} \mathrm{C}$ and $81.26 \%$ at $28^{\circ} \mathrm{C}$ with a 10 minutes shock treatment 15 minutes after fertilization. Hatching efficiency was more successful at the $28^{\circ} \mathrm{C}$ heat shock group. It was concluded that the findings of the study could be used for the production of triploid Black Sea trout stock.
\end{abstract}

Keywords: Erythrocyte size, NOR, Salmo trutta labrax, triploid rate.

\section{GíRiș}

Balık yetiştiriciliğinde temel amaç, mümkün olan en kısa zaman diliminde verimli ve sağlıklı ürünler elde etmektir. $\mathrm{Bu}$ amaçla, son yıllarda alabalık üretiminde triploidizasyon uygulamaları yaygın olarak kullanılmaktadır. Triploid balıklar kısır olduğu için diploid balıklara göre önemli avantajları vardır. Metabolik enerjinin gamet üretimi yerine somatik büyüme için kullanılması, daha fazla biyokütle elde edilmesi, daha kaliteli balık eti üretimi, hastalıklara karşı direncin artması ve doğal popülasyonlara kaçması durumunda genetik ve ekolojik olarak daha az tehdit etmeleri en önemli avantajlardandır (Johnson vd., 2004; Sonay, 2013). Genetik etkinin sınırlı olmasından dolayı birçok uluslararası organizasyon (NASCO, FAO, ICES) 
tarafindan yetiştiricilikte ve balıklandırma uygulamalarında triploidizasyonun kullanılması önerilmektedir. Ayrıca, triploid balıkların Avrupa Birliği mevzuatına göre genetiği değiştirilmiş organizma (GDO) olarak kabul edilmemesi triploid balık üretimini artırmıştır (Yılmaz vd., 2017). Triploid balık üretmek için 1sı şoku (sıcaklık, soğukluk), hidrostatik basınç ve kimyasal uygulamaları gibi çeşitli çevresel şoklar kullanılmaktadır (Felip vd., 1997; Özden vd., 2003). Kahverengi alabalıklarda (Salmo trutta) sıcaklık şoku ile birçok triploidizasyon çalışması yürütülmüş ve bu çalışmalarda şok için uygun sıcaklık ve yaşama oranları belirlenmiştir (Arai \& Wilkins, 1987; Crozier \& Moffett, 1989; Quillet vd., 1991). Ayrıca, Karadeniz alabalığında (Salmo labrax) (Çakmak vd., 2019), kaynak alabalı̆̆ında (Salvelinus fontinalis) (Dubé vd., 1991), Aras alabalığında (Salmo trutta caspius) (Kalbassi vd., 2009), gökkuşağ 1 alabalığında (Dillon, 1988; Happe vd., 1988) sıcaklık şoku uygulanarak triploid balık elde edilmiştir.

Triploid oranını belirlemede; kromozom sayımı veya karyotipleme, nükleolar bölgelerin gümüş nitrat ile boyanmas1 (NOR (Nucleolar Organizer Region)), proteinlerin elektroforezi, çekirdek ve hücre boyutunun ölçümü, eritrosit büyüklüğü ve DNA içeriği yöntemleri kullanılmaktadır (Felip vd., 2001; Bai vd., 2011; Kenanoğlu vd., 2013; Kim vd., 2017).

Karadeniz alabalığı, Doğu Karadeniz'de yayılım gösteren anadrom ve endemik bir salmonid türüdür (Akhan vd., 2011a; Çakmak vd., 2019). Yüksek ekonomik değere sahip olan türün Türkiye'nin Doğu Karadeniz Bölgesi’nde bazı alabalık çiftliklerinde ticari olarak kültürü yapılmaktadır (Canyurt \& Akhan, 2009; Akhan vd., 2011b; Sonay, 2013), ancak kültür koşullarında gökkuşağı alabalığına oranla daha yavaş büyüdüğü için üretim miktarı yeterli düzeye ulaşamamıştır (Aksungur vd., 2005).

Bu çalışmada, triploid Karadeniz alabalı̆̆ı üretimi için sıcaklık şoku derecesi, şok zamanı ve süreleri belirlenerek Karadeniz alabalığının kuluçka randımanı üzerine etkilerinin belirlenmesi amaçlanmıştır.

\section{MATERYAL VE METOT}

Materyal: $\mathrm{Bu}$ çalışmada, $2^{+}$yaşlı damızlık Karadeniz alabalıkları Karadeniz Teknik Üniversitesi Deniz Bilimleri Fakültesi Prof. Dr. İbrahim Okumuş Araştırma Ünitesi'nden temin edilmiştir. Araştırmada ortalama boyu $64,8 \pm 6,2 \mathrm{~cm}(53,9-73,8 \mathrm{~cm})$, ortalama

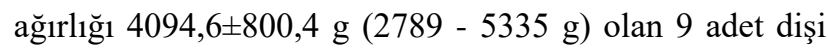
damızlık ile ortalama boyu $62,0 \pm 1,6 \mathrm{~cm}(59,5-63,9 \mathrm{~cm})$ ve ortalama ağırlığ $2731,0 \pm 484,7 \mathrm{~g}$ (2012-3130 g) olan 6 adet erkek damızlık kullanılmıştır. Damızlık balıklar $\% 10$ 'luk benzokain çözeltisinden $0,5 \mathrm{ml} / 1$ kullanılarak bayıltılmıştır (Serezli vd., 2005). Boy-ağırlık verileri alındıktan sonra sağım ve dölleme işlemi gerçekleştirilmiştir. Her bir sıcaklık şok uygulaması için farklı damızlık balıklar kullanılmıştır. Çalışmada kullanılan yumurtaların ortalama çapı $5,6 \pm 0,3 \mathrm{~mm}$ ve kuluçka suyu ortalama $11,3 \pm 0,64(9,9-12,4){ }^{\circ} \mathrm{C}$ olarak belirlenmiştir.

Yumurtaları dölleme işlemi sonrasında her bir grupta ortalama $1080 \pm 344,19$ adet olacak şekilde üç tekerrürlü 16 grup (4 diploid ve 12 triploid $)$ oluşturulmuştur. Kontrol (diploid) grubu $10-12{ }^{\circ} \mathrm{C}$ su sıcaklığında tutulmuş, diğer gruplara ise döllendikten sonra $10,15,20$. dakikalarda $26,28,30$ ve $32{ }^{\circ} \mathrm{C}$ sıcaklık şoku uygulanmıştır. Sicaklık şok süresi tüm triploid gruplarda 10 dakika sürmüştür (Arai \& Wilkins, 1987; Crozier \& Moffett, 1989). Döllülük oranını belirlemek amacıyla 1012 saat sonra 50 adet yumurta örneklenmiş ve glasiyel asetik asit, aseton, saf su (1:1:1) çözeltisinde 3-5 dakika bekletildikten sonra mikroskop altında çekirdek bölünmesi tespit edilmiştir (Serezli, 2004). Kuluçka dolabına yerleştirilen döllenmiş yumurtaların her gün düzenli olarak su sicaklık parametreleri ve ölü yumurta sayıları belirlenmiştir. Kuluçka dönemi süresince elde edilen verilerden döllülük oranı, çıkış oranı ve kuluçka randımanı değerleri belirlenmiştir (Tablo 1) (Hisar vd., 2003; Baki, 2006):

Döllülük oranı (\%)=(Döllü yumurta adedi/ Toplam yumurta adedi) $x 100$ Çıkış oranı $(\%)=($ Canlı yavru adedi/ Döllü yumurta adedi) $\times 100$

Kuluçka randımanı $(\%)=(C ̧ ı k a n$ canlı yavru adedi/ Toplam yumurta adedi) $\mathrm{x} 100$

Sıcaklık şoku uygulaması sonucu elde edilen triploid oranını belirlemek amacıyla iki farklı yöntem (NOR boyama, eritrosit büyüklüğü) kullanılmıştır. NOR bölgesinin gümüş nitrat boyama tekniği besin kesesini tamamen tüketip aktif yüzmeye ve yem almaya başlayan larvalarda ( $\mathrm{n}=50)$ (Howell \& Black, 1980; Saygun, 2005), eritrosit boyama yöntemi ise ortalama diploidlerde boy $4,98 \pm 1,69 \mathrm{~cm}$ ve ağırlık $1,41 \pm 1,19 \mathrm{~g}$, triploidlerde boy $5,57 \pm 0,67 \mathrm{~cm}$ ve ağırlık $1,45 \pm 0,65 \mathrm{~g}$ olan yavrularda $(\mathrm{n}=50)$ gerçekleştirilmiştir (URL 1; Gao vd., 2007; Dorafshan vd., 2008). Eritrositleri boyanan balıkların aynı zamanda NOR oranlarına da bakılarak triploid bireyler kontrol edilmiştir. NOR boyamada her bir balıktan üç tekerrürlü preparatlar hazırlanmış ve her preparattan 1000x büyütmede 50 adet hücre sayılmıştır. Elde edilen verilerden, tek nukleoli içeren hücreler haploid, bir ve iki nukleoli içerenler diploid ve bir, iki, üç ve daha fazla nukleoli içeren hücreler ise triploid olarak kabul edilmiştir (Şekil 1) (Piferrer vd., 2000; Okumura vd., 2001; Jankun vd., 2007). Eritrosit çap ve çekirdek ölçümlerinden ploidiyi belirlemek için her balıktan üç tekerrürlü preparatlar hazırlanmış ve her preparattan 50 eritrosit hücresinin major aksis, minor aksis, çekirdek major aksis ve çekirdek minor aksis ölçümleri yapılmıştır. Elde edilen verilerden; eritrosit yüzey alanı $\left(\mu \mathrm{m}^{2}\right)$, eritrosit hacmi $\left(\mu \mathrm{m}^{3}\right)$, çekirdek yüzey 
alanı $\left(\mu \mathrm{m}^{2}\right)$ ve çekirdek hacmi $\left(\mu \mathrm{m}^{3}\right)$ belirlenmiştir (Tablo 2) (Akhan vd., 2011a; Akhan vd., 2011b; Dorafshan vd., 2008).

Çalışma sonunda verilerin değerlendirilmesi için Excel ve SigmaPilot 11. paket programı kullanılmıştır.
Diploid ve triploid eritrosit ölçümlerinin karşılaştırılması t testi veya Mann Whitney testiyle, grupların karşılaştırılması tek yönlü varyans analizi (one-way ANOVA) ve Tukey çoklu karşılaştırma testi ile değerlendirilmiştir.

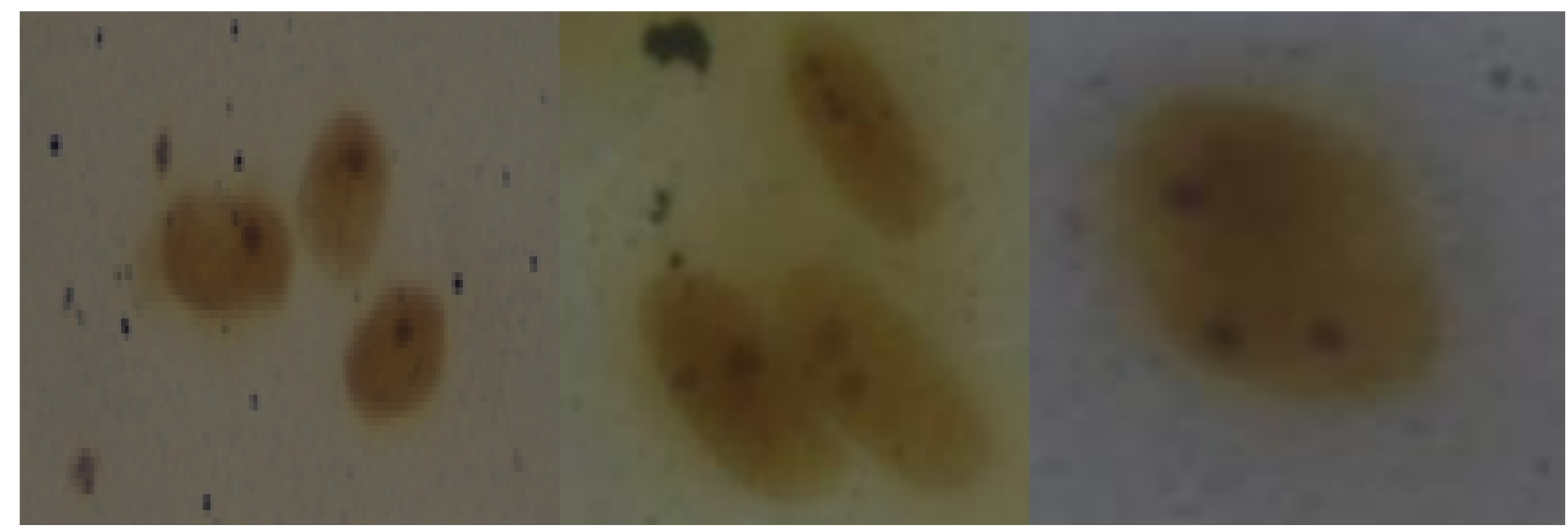

Şekil 1. NOR preperatları (A: tek nukleoli, B:iki nukleoli, C: üç nukleoli).

Figure 1. NOR preparations (A: one nucleoli, B: two nucleoli, C: three nucleoli).

\section{BULGULAR VE TARTIŞMA}

Bu çalışmada dört farklı sıcaklık şoku uygulanan yumurtaların kuluçka performansı ve triploid oranları belirlenmiştir (Tablo 1). Döllenen triploid ve diploid yumurtalarda; gözlenme 220,4 gün-derece (GD), yumurtadan çıkış 386,1 GD ve çıkıştan sonra besin kesesi tüketimi 414,2 GD'de gerçekleşmiştir, gruplar arasında gözlemsel olarak bir fark görülmemiştir. Sıcaklık şoku uygulamasının döllenme oranı üzerine etkisi görülmemiştir. En iyi döllenme oran $128^{\circ} \mathrm{C}$ şok uygulanan triploid deneme grubunun kontrol grubunda belirlenmiştir. Çalışma sonuçları Kankaya, (1998)'nın yaptığı triploid gökkuşağı alabalıklarında sıcaklık şok uygulaması ile benzerlik göstermiş ve şok uygulamasının döllenme oranı üzerine olumsuz etkisi gözlemlenmemiştir. Çıkış oranı ve kuluçka randımanı gruplar arasında farklılıklar göstermiştir $(\mathrm{P}<0,05)$ (Tablo 1). Çıkış oranı ve kuluçka randımanı değerleri diploid gruplarda triploidlerden daha başarılı olmuştur. Karadeniz alabalığında (Akhan vd., 2011b; Çakmak vd., 2019), kahverengi alabalıkta (Quillet vd., 1991; Crozier \& Moffett, 1989; Arai \& Wilkins, 1987), kaynak alabalığı (S. fontinalis) (Dubé vd., 1991), diploid ve triploid salmonid hibritlerde (Gray vd., 1993), gökkuşağı alabalığında (Solar vd., 1984; Kankaya, 1998; Blanc vd., 2000) benzer sonuçları elde etmişlerdir. Triploid gruplarda meydana gelen yaşama oranındaki düşüklük yüksek sıcaklık şoku ve süresi (Kalbassi vd., 2009), yumurta kalitesi veya farklı menşeili yumurtaların triploidizasyon uygulamalarına karşı duyarlı olduğu düşünülmektedir (Arai \& Wilkins, 1987).

Alabalıklarda farklı sıcaklık, şok süresi ve zamanı uygulamaları ile triploid balık üretimi birçok çalışma ile ortaya konulmuştur (Solar vd., 1984; Arai \& Wilkins, 1987; Crozier \& Moffett, 1989; Liu \& Quillet, 1989; Dubé vd., 1991; Ojolick vd., 1995; Kankaya, 1998; Blanc vd., 2000; Kalbassi vd., 2009; Akhan vd. 2011a; Akhan vd., 2011b; Kızak vd., 2013; Çakmak vd., 2019). Bu çalışmada, döllenen yumurtalara dört farklı sıcaklık şoku uygulanmış, en yüksek triploid oran 128 ve $32{ }^{\circ} \mathrm{C}$ 'lerde elde edilmiştir. Ancak $32{ }^{\circ} \mathrm{C}$ grubunda yaşama oranı diğer gruplara göre düşük bulunmuştur. Gruplar arasındaki triploid oranı değerleri $28{ }^{\circ} \mathrm{C}$ ve $32{ }^{\circ} \mathrm{C}$ sıcaklık şoku uygulamalarında istatistiksel farkl11.k $(\mathrm{P}<0,05), \quad 26^{\circ} \mathrm{C}$ ve $30^{\circ} \mathrm{C}$ uygulamalarında ise benzerlik göstermiştir. $28^{\circ} \mathrm{C}$ döllenme işleminden 10 dakika sonra 10 dakika sıcaklık şoku ile $\% 79,26 \pm 1,05$ ve döllenme işleminden 15 dakika sonra 10 dakika sıcaklık şoku ile $\% 81,26 \pm 3,94$ triploid oranı elde edilmiştir $(\mathrm{P}<0,05)$ (Tablo 1). Kahverengi alabalıklarda 28 ${ }^{\circ} \mathrm{C}$ de triploid oranını; Quillet vd., (1991) \%84,2-94,7 olarak, Crozier \& Moffett, (1989) \%60-100 ve Kalbassi vd., (2009) \%27-84 olarak bildirmiştir.

Ploidy oranını belirlemek amacıyla iki farklı yöntem (NOR boyama ve eritrosit ölçümü) kullanılmıştır. NOR boyama tekniği larval safhadan damızlık boyuta kadar farklı büyüklüklerde uygulanabilen basit, ucuz ve kısa sürede ploidi oranını belirlemede kullanılan yöntemlerden bir tanesidir. NOR bölgeleri $\mathrm{AgNO}_{3}$ ile boyanmaktadır (Felip vd., 1997; Piferrer vd., 2000; Gündoğdu, 2016). NOR boyama yöntemi salmonidlerde (Lozano vd., 1992; Jankun vd., 2007), kalkanda (Scophthalmus maximus) (Piferrer vd., 2000; Piferrer vd., 2003), levrekte (Dicentrarchus labrax), (Sola vd., 1993; Felip vd., 1997), ve turna balığında (Esox lucius) (Jankun vd., 1998) uygulanmıştır. Bu çalışmada, NOR lokasyonları incelenerek hücrelerin nukleolileri sayılarak ortalamaları 
alınmıştır. Diploid hücrelerin nukleoli ortalaması 1,10 ile 1,96 arasında belirlenirken, triploid hücrelerin nukleoli ortalaması 1,60 ile 2,83 arasında değişmiştir. Ortalama 1,52 ile 1,96 arasında ploidy oranlarında belirsizlik görüldüğünden dolayı yavru balıklardan örnekleme yapılmış NOR lokasyonları ve eritrosit çapları da kullanılarak ortalama NOR $>1,80$ olan bireyler triploid, diğerleri ise diploid olarak kabul edilmiştir. Ortalama nukleoli sayısı triploidlerde diploidlerden önemli ölçüde daha yüksektir. Kalkan balıklarında (S. maximus) ploidi oranının belirlenmesinde kullanılan NOR boyama yöntemi sonucu; 1,10 ile 1,85 arasında olanları diploid, 1,50 ile 2,35 arasında olanları triploid olarak bildirmiş, fakat 1,50 ile 1,85 arasında diploid ve triploidlerin çakışmasından dolayı NOR $>1,735$ olanları triploid olarak kabul etmiştir (Piferrer vd., 2000). Salmonlarda (Oncorhynchus mykiss, O. kisutch) ortalama nukleoli ortalaması triploidlerde 2,75 olarak belirlenirken diploidlerde 1,63 olarak belirlenmiştir (Lozano vd., 1992).

Tablo 1. 26, 28, 30 ve $32^{\circ} \mathrm{C}$ 'lerde uygulanan sıcaklık şok zamanı, şok süresi, triploid oranı, döllenme oranı, çıkış oranı ve kuluçka randımanı verileri. Table 1. Data of heat shock time, shock duration, triploid rate, fertilization rate, hatching rate and hatching efficiency at $26,28,30$, and $32^{\circ} \mathrm{C}$.

\begin{tabular}{|c|c|c|c|c|c|c|}
\hline $\begin{array}{c}\text { Şok sıcaklığ } \\
\left({ }^{\circ} \mathrm{C}\right)\end{array}$ & $\begin{array}{l}\text { Şok süresi } \\
\text { (Dakika) }\end{array}$ & $\begin{array}{c}\text { Döllenme sonrası şok zamanı } \\
\text { (Dakika) }\end{array}$ & $\begin{array}{c}\text { Triploid oranı } \\
(\%)\end{array}$ & $\begin{array}{c}\text { Döllenme oranı } \\
(\%)\end{array}$ & $\begin{array}{c}\text { Çıkış oranı } \\
(\%)\end{array}$ & $\begin{array}{c}\text { Kuluçka randımanı } \\
(\%)\end{array}$ \\
\hline Kontrol & - & - & 0 & $78,33 \pm 2,88$ & $75,82 \pm 7,72^{\mathrm{a}}$ & $56,01 \pm 5,31^{\mathrm{a}}$ \\
\hline 26 & 10 & 10 & $66,67 \pm 3,09$ & $80,00 \pm 5,00$ & $47,35 \pm 7,56^{\mathrm{b}}$ & $30,22 \pm 8,16^{\mathrm{b}}$ \\
\hline 26 & 10 & 15 & $63,22 \pm 5,28$ & $75,00 \pm 5,00$ & $46,93 \pm 8,19^{b}$ & $29,06 \pm 6,99^{b}$ \\
\hline 26 & 10 & 20 & $41,33 \pm 2,26$ & $76,68 \pm 2,89$ & $64,92 \pm 5,72^{\mathrm{a}}$ & $46,89 \pm 2,49^{\mathrm{a}}$ \\
\hline Kontrol & - & - & 0 & $86,68 \pm 2,89$ & $81,87 \pm 3,59^{\mathrm{a}}$ & $64,37 \pm 0,68^{\mathrm{a}}$ \\
\hline 28 & 10 & 10 & $79,26 \pm 1,05^{\mathrm{a}}$ & $81,68 \pm 2,89$ & $49,48 \pm 10,22^{b}$ & $39,44 \pm 7,01^{\mathrm{b}}$ \\
\hline 28 & 10 & 15 & $81,26 \pm 3,94^{\mathrm{a}}$ & $78,33 \pm 2,89$ & $46,75 \pm 11,73^{b}$ & $36,19 \pm 8,07^{\mathrm{b}}$ \\
\hline Kontrol & - & - & 0 & $80,00 \pm 5,00$ & $70,74 \pm 6,64^{\mathrm{a}}$ & $30,98 \pm 3,97^{\mathrm{a}}$ \\
\hline 30 & 10 & 10 & $72,96 \pm 4,60$ & $78,33 \pm 2,89$ & $28,32 \pm 6,09^{\mathrm{b}}$ & $20,02 \pm 4,06^{\mathrm{ab}}$ \\
\hline 30 & 10 & 15 & $52,52 \pm 6,39$ & $83,33 \pm 2,89$ & $19,59 \pm 12,40^{\mathrm{b}}$ & $12,72 \pm 7,66^{\mathrm{b}}$ \\
\hline 30 & 10 & 20 & $59,35 \pm 3,52$ & $78,33 \pm 5,77$ & $14,81 \pm 11,29^{b}$ & $9,26 \pm 9,12^{\mathrm{b}}$ \\
\hline Kontrol & - & - & 0 & $83,33 \pm 5,77$ & $66,40 \pm 6,14^{\mathrm{a}}$ & $43,20 \pm 3,50^{\mathrm{a}}$ \\
\hline 32 & 10 & 10 & $62,73 \pm 11,06^{\mathrm{a}}$ & $85,00 \pm 5,00$ & $14,47 \pm 9,89^{b}$ & $10,61 \pm 7,16^{\mathrm{b}}$ \\
\hline 32 & 10 & 15 & $86,15 \pm 8,74^{\mathrm{b}}$ & $81,67 \pm 2,89$ & $16,09 \pm 16,29^{b}$ & $19,15 \pm 12,02^{\mathrm{b}}$ \\
\hline 32 & 10 & 20 & $74,08 \pm 5,43^{\mathrm{c}}$ & $81,67 \pm 5,77$ & $19,84 \pm 7,39^{\mathrm{b}}$ & $18,31 \pm 4,18^{\mathrm{b}}$ \\
\hline
\end{tabular}

NOR boyama ile elde edilen verilerin kontrol edilmesi amaciyla yavru balıklardan alınan kan örneklerinin eritrosit büyüklüklerinin ölçümleri gerçekleştirilmiştir. Triploid ve diploid Karadeniz alabalıklarının eritrosit ve çekirdek büyüklükleri ölçülmüş, elde edilen verilerden eritrosit ve çekirdek yüzey alanı ve hacmi hesaplanmıştır (Tablo 2). Triploid balıklara ait eritrosit ve çekirdek bulguları diploidlerden daha yüksek değerlerde hesaplanmıştır $(\mathrm{P}<0,001)$ (Tablo 2). Triploid balıkların eritrosit büyüklükleri, hücre hacimleri ve yüzey alanları diploid balıklardan daha büyük olduğu birçok araştırmacı tarafindan ortaya konulmuştur. Karadeniz alabalığında (Akhan vd., 2011a; Çakmak vd., 2019), kaynak alabalığında (S. fontinalis) (Woznicki \& Kuzminski, 2002), Aras alabalığında (S.t. caspius) (Dorafshan vd., 2008), kalkan balığında (S. maximus) (Cal vd., 2005), levreklerde (D. labrax) (Peruzzi vd., 2005), Sibirya mersin balığında (Acipenser baeri) (Dorota vd., 2006), tilapia balığında (Tilapia aurea) (Valenti, 1975) ve kanal yayın balığında (Ictalurus punctatus) (Wolters vd., 1982) yapılan çalışmalarda triploidler diploidlerden daha yüksek eritrosit büyüklüğüne sahip olduğu belirlenmiştir.

Tablo 2. Triploid ve diploid bireylerde eritrosit ve çekirdeklere ait veriler.

Table 2. Data of erythrocytes and nucleus in triploid and diploid individuals.

\begin{tabular}{lccc}
\hline & Triploid & Diploid & Oran (T/D) \\
\hline Eritrosit minor aksis $(\mu \mathrm{m})$ & $10,93 \pm 0,06$ & $7,58 \pm 0,09$ & 1,44 \\
Eritrosit major aksis $(\mu \mathrm{m})$ & $17,08 \pm 0,16$ & $14,66 \pm 0,86$ & 1,16 \\
Eritrosit yüzey alan $\left(\mu \mathrm{m}^{2}\right)$ & $146,99 \pm 6,41$ & $87,25 \pm 1,20$ & 1,68 \\
Eritrosit hacmi $\left(\mu \mathrm{m}^{3}\right)$ & $1074,75 \pm 28,37$ & $446,45 \pm 12,87$ & $<001$ \\
Çekirdek minor aksis $(\mu \mathrm{m})$ & $5,10 \pm 0,34$ & $4,00 \pm 0,03$ & $<, 001$ \\
Çekirdek major aksis $(\mu \mathrm{m})$ & $8,01 \pm 0,04$ & $7,05 \pm 0,05$ & 1,28 \\
Çekirdek yüzey alanı $\left(\mu \mathrm{m}^{2}\right)$ & $32,29 \pm 2,43$ & $22,16 \pm 0,15$ & 1,14 \\
Çekirdek hacmi $\left(\mu \mathrm{m}^{3}\right)$ & $112,56 \pm 7,46$ & $59,05 \pm 1,90$ & $<001$ \\
\hline
\end{tabular}

Mayotik triploid balık üretimi; yumurtaların döllenmesinden hemen sonra çevresel şok (sıcaklık şoku, basınç, kimyasallar) uygulanarak, mayoz bölünme bloke edilip, ikinci kutup hücresinin döllenmeden sonra tutulması ile gerçekleşmektedir. Şok uygulamasının zamanı ve süresi farklı balık türlerinde triploid oranını etkilemektedir.

\section{SONUÇ}

Balık yetiştiriciliğinde triploidizasyon uygulanabilir bir üretim yöntemidir. Üreticiler daha kısa 
sürede fazla ürün elde etmek için triploid balık üretimini tercih etmektedir. Fakat triploid balık üretimi bazı zorluklar içermektedir. Sıcaklık şoku uygulaması ile triploid balık elde edilmesi en fazla kullanılan ve en pratik uygulanabilir yöntemdir. Sağlıklı bir triploidizasyon uygulaması için balık türüne göre uygun zamanda ve uygun sıcaklıkta uygulama yapmak esastır. Bu çalışmada Karadeniz alabalığı olarak bilinen Salmo trutta labrax türünde sıcaklık şoku ile triploid uygulaması çalışılarak, uygun sıcaklık ve uygulama zamanları belirlenmiştir. Sonuç olarak, triploid Karadeniz alabalıklarında döllenmiş yumurtalara uygulanacak sicaklık şokunun döllenme işleminden 15 dakika sonra $28^{\circ} \mathrm{C}$ 'de 10 dakika uygulama şeklinde yapılması durumunda en ideal döllenme ve yaşama oranları elde edilmiştir. Elde edilen sonuçlar, ülkemiz endemik türü olan Karadeniz alabalığı triploid uygulamalarında kullanılabilir. Ayrıca, triploid birey oranını belirlemede hılı, kolay ve en yaygın olarak kullanılan eritrosit ölçümü yanı sıra, NOR boyama yönteminin erken safhada (larval safhada) ploidi oranını belirlemede kullanılabileceği de bu çalışma ile ortaya konulmuştur.

\section{TEŞEKKÜR}

$\mathrm{Bu}$ çalışma doktora tezinden üretilmiş ve Karadeniz Teknik Üniversitesi BAP tarafından 2009.117.001.5 nolu proje ile desteklenmiştir.

\section{KAYNAKLAR}

Akhan, S., Serezli, R. \& Delihasan Sonay, F. (2011a). Hematology of diploid and triploid rainbow trout (Oncorhynchus mykiss), Black Sea trout (Salmo labrax Pallas, 1814) and their $\mathrm{F}_{1}$ hybrids. The Israeli Journal of Aquaculture, Bamidgeh, 63, 4.

Akhan, S., Delihasan Sonay, F., Okumus, I., Köse, Ö. \& Yandi, I. (2011b). Inter-specific hybridization between Black Sea trout (Salmo labrax Pallas, 1814) and rainbow trout (Oncorhynchus mykiss Walbaum, 1792). Aquaculture Research, 42, 1632-1638. DOI: $\quad 10.1111 / \mathrm{j} .1365-$ 2109.2010.02755.x

Aksungur, M., Yılmaz, C., Tabak, İ., Aksungur, N. \& Misır, D.S. (2005). Karadeniz alabalığ (Salmo trutta labrax, 1811)'nın kültür şartlarına adaptasyonu. Fırat Üniversitesi, Fen ve Mühendislik Bilimleri Dergisi, 17, 349-359.

Arai, K. \& Wilkins, N.P. (1987). Triploidization of brown trout (Salmo trutta) by heat shocks. Aquaculture, 64, 97-103. DOI: 10.1016/00448486(87)90345-0

Bai, Z., Liu, F., Li, J. \& Yue, G.H. (2011). Identification of triploid individuals and clonal lines in Carassius auratus complex using microsatellites. International Journal of
Biological Sciences, 7(3), 279-285. DOI: 10.7150/ijbs.7.279

Baki, B. (2006). Gökkuşă̆l alabalıklarından (Oncorhynchus mykiss, W., 1792) elde edilen yumurtaların iki farklı su kaynă̆ında açılma süreleri, larva çıkışı ve büyümelerinin karşılaştırılması. Doktora Tezi, Ondokuz Mayıs Üniversitesi Fen Bilimleri Enstitüsü, Samsun, Türkiye, 88s.

Blanc, J.M., Vallée, F. \& Dorson, M. (2000). Survival, growth and dressing traits of triploid hybrids between rainbow trout and three charr species, Aquaculture Research, 31, 349-358. DOI: 10.1046/j.1365-2109.2000.00446.x

Cal, R.M., Vidal, S., Camacho, T., Piferrer, F. \& Guitian, F.J. (2005). Effect of triploidy on turbot haematology, Comparative Biochemistry and Physiology, Part A: Molecular and Integrative Physiology, 141, 35-41. DOI: 10.1016/j.cbpb.2005.03.007

Canyurt, M.A. \& Akhan, S. (2009). Development and situation of trout culture in Turkey. pp. 90-94. In: 15th Annual 15th International Scientific Conference Proceedings: Research for Rural Development 2009. 20-22 May 2009, Jelgava, Latvia.

Crozier, W.W. \& Moffett, I.J.J. (1989). Experimental production of triploid brown trout, Salmo trutta L., using heat shock. Aquaculture \& Fisheries Management, 20, 343-353. DOI: 10.1111/j.1365-2109.1989.tb00362.x

Çakmak, E., Çankırılıgil, E.C., Düzgüneş, Z.D., Özel, O.T., Eroğlu, O. \& Firidin, Ş. (2019). Triploid Black Sea trout (Salmo labrax Pallas, 1814) induced by heat shock and evaluation of triploidy with different techniques. Genetics of Aquatic Organisms, 3(1), 01-07. DOI: 10.4194/24591eticf 831-v3_1_01

Dillon, J.C. (1988). Production of triploid rainbow trout for evaluation in South Dakota waters. The Degree Master of Science, South Dakota State University, Wildlife and Fisheries Sciences. South Dakota. 69 p.

Dorafshan, S., Kalbassi, M.R., Pourkazemi, M., Amiri, B.M. \& Karimi, S.S. (2008). Effects of triploidy on the Caspian salmon Salmo trutta caspius haematology. Fish Physiology \& Biochemistry, 34, 195-200.

Dorota, F.B., Jankun, M. \& Wosnicki, P. (2006). Chromosome number and erythrocyte nuclei length in triploid Siberian sturgeon Acipenser baeri Brandt. Caryologia, 59 (4), 319-321. DOI: 10.1080/00087114.2006.10797932

Dubé, P., Blanc, J.M., Chouinard, M. \& Noüe, J. (1991). Triploidy induced by heat shock in brook trout (Salvelinus fontinalis). Aquaculture, 92, 305-311. DOI: 10.1016/00448486(91)90036-7

Felip, A., Zanuy, S., Carrillo, M. \& Piferrer, F. (2001). Induction of triploidy and gynogenesis in teleost fish with emphasis on marine species. 
Genetica, 111, 175-195. DOI: 10.1023/a:1013724322169

Felip, A., Zanuy, S., Carrillo, M., Martinez, G., Ramos, J. \& Piferrer, F. (1997). Optimal conditions for the induction of triploidy in the sea bass (Dicentrarchus labrax L.), Aquaculture, 152, 287-298. DOI: 10.1016/S0044-8486(96)01509-8

Gao, Z., Wang, W., Abbas, K., Zhou, X., Yang, Y., Diana, J.S., Wang, H., Wang, H., Li, Y. \& Sun, Y. (2007). Haematological characterization of loach Misgurnus anguillicaudatus: Comparison among diploid, triploid and tetraploid specimens. Comparative Biochemistry \& Pysiology, Part A: Molecular \& Integrative Physiology, 147, 1001-1008. DOI: $10.1016 /$ j.cbpa.2007.03.006

Gray, A.K., Evans, M.A. \& Thorgaard, G.H. (1993). Viability and development of diploid and triploid salmonid hybrids. Aquaculture, 112, 125-142. DOI: 10.1016/0044-8486(93)90439-6

Gündoğdu, H. (2016). Endemik Beyşehir kababurun baliğl, Chondrostoma beysehirense (Bogutskaya, 1997) üzerine sitogenetik araştırmalar. Yüksek Lisans Tezi, Selçuk Üniversitesi Fen Bilimleri Enstitüsü. Konya, Türkiye, $43 \mathrm{~s}$.

Happe, A., Quillet, E. \& Chevassus, B. (1988). Early life history of triploid rainbow trout (Salmo gairdneri Richardson). Aquaculture, 71, 107118. DOI: 10.1016/0044-8486(88)90278-5

Hisar, S.A., Yanık, T. \& Hisar, O. (2003). Hatchery and growth performance of two trout pure breeds, Salvelinus alpinus and Salmo trutta fario, and their hybrid. The Israeli Journal of Aquaculture-Bamidgeh, 55(3), 154-159. DOI: 10.46989/001c.20357

Howell, W.M. \& Black, D.A. (1980). Controlled silver-staining of nucleolar organizer regions with a protective colloidal developer: A 1-step Method. Experienta, 36, 1014-1015. DOI: 10.1007/BF01953855

Jankun, M., Kuzminski, H. \& Furgala-Selezniow, G. (2007). Cytologic ploidy determination in fishan example of two salmonid species. Environmental Biotechnology, 3(2), 52-56.

Jankun, M., Woznicki, P., Dajnowicz, G., DemskaZakes, K., Luczynski, M.J. \& Luczynski, M. (1998). Heterochromatin and NOR location in Northern Pike (Esox lucius), Aquatic Science, 60, 17-21.

Johnson, R.M., Shrimpton, J.M., Heath, J.W. \& Heath, D.D. (2004). Family, induction methodology and interaction effects on the performance of diploid and triploid chinook salmon (Oncorhychus tshawytscha). Aquaculture, 234, 123-142.

Kalbassi, M.R., Dorafshan, S., Pourkazemi, M. \& Amiri, B.M. (2009). Triploidy induction in the Caspian Salmon, Salmo trutta caspius, by heat shock. Journal of Applied Ichthyology, 25,
104-107. DOI: 0426.2008.01177.x

Kankaya, E. (1998). Gökkuşağı alabalığında (Oncorhynchus mykiss W., 1792) isı şoku uygulamasıyla triploidi oluşturulması üzerine bir araştırma. Yüksek Lisans Tezi, Yüzüncü Y1l Üniversitesi, Fen Bilimleri Enstitüsü, 53s.

Kenanoğlu, O.N., Yilmaz, S., Ergun, S. \& Aki, C. (2013). A preliminary study on the determination of triploidy by chromosome analysis at the different stages of development in rainbow trout, Oncorhynchus mykiss. Marine Science \& Technology Bulletin, 2(2), 17-21.

Kızak, V., Güner, Y., Türel, M. \& Kayım, M. (2013). Comparison of growth performance, gonadal structure and erythrocyte size in triploid and diploid brown trout (Salmo trutta fario L, 1758). Turkish Journal of Fisheries \& Aquatic Sciences, 13, 571-580. DOI: 10.4194/1303-2712-v13_4_02

Kim, H.S., Chung, K.H. \& Son, J.H. (2017). Comparison of different ploidy detection methods in Oncorhynchus mykiss, the rainbow trout. Fisheries \& Aquatic Sciences, 20, 29. DOI: $10.1186 / \mathrm{s} 41240-017-0074-8$

Liu, F.G. \& Quillet, E. (1989). Preliminary results on triploidy induced by heat shocks in the brown trout (Salmo trutta). Journal of the Fisheries Society of Taiwan (JFST). 16(2), 91-95.

Lozano, R., Rejón, C.R. \& Rejón, M.R. (1992). A comparative analysis of NORs in diploid and triploid salmonids: implications with respect to the diploidization process occurring in this fish group. Heredity. 69, 450-457.

Ojolick, E.J., Cusack, R., Benfey, T.J. \& Kerr, S.R. (1995). Survival and growth of all-female diploid and triploid rainbow trout (Oncorhynchus mykiss) reared at chronic high temperature. Aquaculture, 131, 177-187. DOI: 10.1016/0044-8486(94)00338-O

Okumura, S.I., Furukawa, S., Kawai, T., Takahashi, S. \& Yamamori, K. (2001). Comparison of nucleoli number in diploid and triploid larva of Pacific abalone Haliotis discus hannai. Fisheries Science, 67, 176-178. DOI: 10.1046/j.1444-2906.2001.00217.x

Özden, O., Güner, Y. \& Kızak, V. (2003). Tatlısu balık kültüründe uygulanan bazı biyoteknolojik yöntemler. Ege Üniversitesi, $S u$ Ürünleri Dergisi, 20(3-4), 563-574.

Peruzzi, S., Varsamos, S., Chatain, B., Fauvel, C., Menu, B., Falguière, C., Sévère, A. \& Flik, G. (2005). Haematological and physiological characteristics of diploid and triploid sea bass, Dicentrarchus labrax L. Aquaculture, 244(1-4), 359-367. DOI: 10.1016/j.aquaculture.2004.11.028

Piferrer, F., Cal, R.M., Àlvarez-Blázquez, B., Sánchez, L. \& Martinez, P. (2000). Introduction of triploidy in the turbot (Scophthalmus maximus) I. Ploidy determination and the effects of cold 
shocks. Aquaculture, 188, 79-90. DOI: 10.1016/S0044-8486(00)00306-9

Piferrer, F., Cal, R.M, Gómez, C., Bouza, C. \& Martinez, P. 2003. Introduction of triploidy in the turbot (Scophthalmus maximus) II. Effects of cold shock timing and induction of triploidy in a large volume of eggs, Aquaculture, 220, 821-831. DOI: 10.1016/S0044-8486(02)00535-5

Quillet, E., Foisil, L., Chevassus, B., Chourrout, D. \& Liu, F.G. (1991). Production of all-triploid and all-female brown trout for aquaculture. Aquatic Living Resources, 4, 27-32. DOI: 10.1051/alr:1991002

Saygun, S. (2005). Karadeniz'de yaşayan çeşitli yassı balıkların (Pisces, Pleuronectiformes) kromozom yapılarının karşılaştırılması. Doktora Tezi, On Dokuz Mayıs Üniversitesi Fen Bilimleri Enstitüsü. Samsun, Türkiye, 130s.

Serezli, R. (2004). Doğu Karadeniz Bölgesi'nde gökkuşă̆l alabalığı (Oncorhynchus mykiss) damızlık stoklarının să̆ım zamanı, damızlık performansı ve kuluçka randımanlarının belirlenmesi. Doktora Tezi, Karadeniz Teknik Üniversitesi Fen Bilimleri Enstitüsü. Trabzon, Türkiye, 104s.

Serezli, R., Okumuş, I. \& Akhan, S. (2005). Akuakültürde anestezinin kullanımı. Türk Sucul Yaşam Dergisi, 4, 475-480.

Sola, L., Bressanello, S., Rossi, A.R., Iaselli,V., Crosetti, D. \& Cataudella, S. (1993). A karyotype analysis of the genus Dicentrarchus by different staining techniques, Journal of Fish Biology, 43, 329-337. DOI: 10.1111/j.1095-8649.1993.tb00567.x

Solar, I.I., Donaldson, E.M. \& Hunter, G.A. (1984). Induction of triploidy in rainbow trout (Salmo gairdneri RICHARDSON) by heat shock, and investigation of early growth. Aquaculture, 42, 57-67. DOI: 10.1016/0044-8486(84)90313-2

Sonay, F. (2013). Triploid Karadeniz alabalı̆̆ (Salmo trutta labrax Pallas, 1811) üretimi ve büyüme potansiyeli ve et kalitesinin belirlenmesi. Doktora Tezi, Karadeniz Teknik Üniversitesi Fen Bilimleri Enstitüsü. Trabzon, Türkiye, $137 \mathrm{~s}$.

URL

1.

(2012).

http://ressources.ciheam.org/om/pdf/b63/008009 17pdf (22 Eylül 2012).

Valenti, R.J. (1975). Induced polyploidy in Tilapia aurea (Steindachner) by means of temperature shock treatment. Journal of Fish Biology, 7, 519-528. DOI: 10.1111/j.1095-8649.1975.tb04627.x

Wolters, W.R., Chrisman, C.L. \& Libey, G.S. (1982). Erythrocyte nuclear measurement of diploid triploid channel catfish, Ictalurus punctatus (Rafinesque). Journal of Fish Biology, 20, 253258. DOI: $10.1111 / \mathrm{j} .1095-8649.1982 . t b 04706 . x$

Woznicki, P. \& Kuzminski, H. (2002). Chromosome number and erythrocyte nuclei length in triploid brook trout (Salvelinus fontinalis). Caryologia, 55
(4), 295-298. DOI:

10.1080/00087114.2002.10797879

Yılmaz, S., Çelik, E.Ş., Kenanoğlu, O.N. \& Ergün, S. (2017). Asidik stresin triploid ve diploid gökkuşağı alabalığı (Oncorhynchus mykiss)'nın hematolojik, immunolojik ve biyokimyasal kan parametreleri üzerine etkileri. Alınteri Journal of Agricultural Sciences, 32(2),17-24. DOI: 10.28955/alinterizbd.320262 